\title{
La competencia de aprender a aprender en el marco de la educación superior
}

\section{Learning to learn in higher education}

\author{
Laia Lluch Molins \\ María Cinta Portillo Vidiella \\ Universidad de Barcelona (UB), España
}

\begin{abstract}
Resumen
La finalidad del artículo es el análisis de la competencia de aprender a aprender, sus dimensiones, factores asociados, metodologías y sistemas de evaluación para su implementación en la educación superior. La incorporación del enfoque competencial en el EEES se ha caracterizado, preferentemente, por el cambio de paradigma que otorga el protagonismo del aprendizaje al alumnado, situándolo en el centro del proceso y valorando el potencial de la evaluación para gestionarlo. En este contexto, aprender a aprender es clave para la educación, en cualquiera de los entornos posibles de aprendizaje.

La aproximación metodológica seguida se basa en la obtención de evidencias científicas desde el análisis documental y de investigaciones recientes con alumnado universitario, a la vez que se profundiza en las propuestas metodológicas y evaluativas que contribuyen al desarrollo de esta competencia. Como resultado, ha sido posible identificar el valor añadido de esta competencia; poner de relieve los retos asociados con su definición y medición; y valorar procesos de evaluación formativa sostenibles que fomentan la toma de conciencia mediante la autoevaluación y la retroalimentación entre iguales, para optimizar el perfil competencial del alumnado universitario. En conclusión, la asunción de aprender a aprender como competencia básica conlleva aprender a centrar la atención en el aprendizaje como actitud vital, que acompaña a la persona de forma permanente. Esto implica cambios en la formación docente, puesto que este reto pide no sólo una constante actualización científica del saber, sino la activación de metodologías que integren la evaluación como proceso continuo de construcción de conocimiento.
\end{abstract}

Palabras clave: autoevaluación; competencia de aprender a aprender; educación superior; evaluación; retroalimentación; toma de conciencia.

\begin{abstract}
The purpose of this article is to analyze the competence of learning to learn, its dimensions, associated factors, methodologies, and evaluation systems for its implementation in higher education. The incorporation of the competence approach in the EHEA has been characterized, preferably, by the paradigm that focus on the importance of learning to the students, placing the student in the center of the process, and valuing the potential of the evaluation to manage it. In this context, learning to learn is key to the education, in any of the possible learning environment.

The methodological approach followed is based on obtaining scientific evidences from the documentary analysis and recent research with university students, while it's analyzed the methodological and evaluative proposals that contribute to the development of the competence to learn to learn. As a result, is has been possible to identify the added value of this competence; to highlight the challenges associated with its definition and measurement; and to evaluate and propose sustainable formative assessment processes that promote awareness through self-assessment and peer feedback to optimize the
\end{abstract}


competences profile of the university students. In conclusion, the assumption of learning to learn as a basic competence entails to learn to focus attention on learning as a vital attitude, which accompanies the person permanently. This implies changes in teacher training, since this challenge demands not only a constant scientific update of knowledge, but also the activation of methodologies that integrate assessment as a continuous process of knowledge construction.

Keywords: assessment; awareness; feedback; higher education; learning to learn; selfassessment.

\section{INTRODUCCIÓN}

Pese al interés mostrado por la comunidad científica a principios de siglo XXI, "la experiencia internacional en investigación y evaluación de la competencia de aprender a aprender es todavía limitada" (Gobierno Vasco, 2012, p. 13). En el Lisbon European Council, se detallaron dos caminos: uno que consistía en investigar qué competencias y conocimientos son necesarios para la sociedad del conocimiento y para el aprendizaje permanente (lifelong learning), y el otro en encontrar maneras de monitorear cómo estas habilidades y conocimientos se desarrollan a través de la educación.

Asimismo, la incorporación al EEES sitúa al estudiante en el centro del proceso como agente activo y protagonista de su aprendizaje, da una oportunidad al impulso del desarrollo de competencias de los universitarios, y la asunción de cambios con respecto a la cultura docente y en la formación del profesorado. Por ello, es interesante delimitar y establecer lo que los estudiantes han de demostrar y saber especificando las competencias, entendiendo competencia como indica DeSeCo, como algo más que conocimiento y habilidades, precisando que las competencias son solo observables en acciones específicas (DESECO-OCDE, 2002). A partir de estas lanzaderas, junto con el Proyecto Tuning Educational Structures in Europe (2002), se definieron los tipos de competencias que más tarde se analizaron y se desarrollaron en los "libros blancos" de cada titulación, según transversales o generales y específicas.

Aunque los objetivos y las competencias son temas complejos para algunos docentes (Zabalza, 2005), "el diseño curricular de un plan de estudios y de sus correspondientes asignaturas debería construirse desde una perspectiva integrada y global" (Riesco, 2008, p. 93), contemplando "las realizaciones o desempeños concretos y con determinados niveles de ejecución o de logro que evidencian lo que el estudiante es capaz de hacer y de demostrar" (Ibarra \& González, 2015, p. 18), y las actitudes académicas y profesionales que debe conseguir como resultados de aprendizaje. 
Con la necesidad de modificar los planteamientos metodológicos, la mayoría de universidades europeas ha ido incorporando en los últimos años el desarrollo competencial en sus planteamientos curriculares, provocando, así, modificaciones en la evaluación "en todos sus niveles, desde un nuevo prisma que la sitúe como una parte fundamental del propio proceso de aprendizaje orientado al desarrollo competencial" (Mateo \& Vlachopoulos, 2013, p. 187). Es imprescindible situar esa competencia como un resultado de aprendizaje a obtener (con diversos niveles competenciales asociados a cada curso), puesto que "parece no ser suficiente planificar la enseñanza esperando que dicha competencia se promueva por el hecho de estudiar o de realizar las actividades de aprendizaje propuestas" (Cano, 2014).

Por todo ello, aprender a aprender se identifica en muchos contextos como una de las habilidades básicas para el éxito en la sociedad del conocimiento (Council European, 2006); de ahí que la finalidad principal de este artículo sea el análisis de la competencia de aprender a aprender, sus dimensiones, factores asociados, metodologías y sistemas de evaluación para su implementación en educación superior.

\section{MARCO TEÓRICO}

La competencia de aprender a aprender emerge de la concepción socio-constructivista de la enseñanza y el aprendizaje, la cual sustenta de modo preferente el enfoque de la enseñanza por competencias. Desde esta concepción, se concibe el aprendizaje como un proceso de construcción de significados y de atribución de sentido mediante la modificación de esquemas de conocimiento. Aprender, en situaciones escolares educativas, se considera que consiste en atribuir significado y sentido a las experiencias y a los contenidos escolares. Significado y sentido son indisociables en la experiencia de construcción de conocimiento y esto es posible en determinadas condiciones; requiere que el sujeto pueda establecer relaciones substantivas, no arbitrarias, entre el conocimiento previo, es decir, el conocimiento disponible en su estructura cognoscitiva -conceptos, procedimientos y actitudes, expectativas, motivaciones, intereses, autoconcepto académico, autoestima, etc.-y el nuevo conocimiento. Solo conectando con el conocimiento previo, no bloqueado por ningún factor, de tipo emocional, por ejemplo, podrá fructificar el nuevo. La estructura mental del aprendiz es el conjunto de esquemas de conocimiento interrelacionados, la representación que tiene una persona en un momento determinado de su historia sobre una parcela de la realidad. Este proceso no se puede desligar de la intervención docente y de su ayuda pedagógica contingentemente ajustada (Coll, 1988, 1995). 
Desde el constructivismo social, para Vygotsky (1978), el aprendizaje es una actividad vinculada necesariamente a un contexto. Supone la participación en una comunidad y no debe limitarse, por tanto, a la adquisición del conocimiento de forma aislada e individualizada, sino a través de la participación social y gracias a la mediación instrumental. Así pues, su optimización recae en la calidad y en la pertinencia de la mediación social, concibiéndola como aquellas interacciones situadas y ajustadas de la persona aprendiz con otra u otras personas expertas, en grados diversos, para la realización de tareas (conceptuales, procedimentales, actitudinales). La búsqueda de ayuda, el apoyo que pueden prestar los iguales, grupo y profesorado, y el trabajo colaborativo con otros, mediante el uso del lenguaje oral y escrito, son factores que definen la dimensión social del aprender a aprender, según Coll, Mauri y Rochera (2012). El proceso de enseñanza-aprendizaje mediado socialmente requiere de constantes ajustes de la ayuda pedagógica para situarse de forma pertinente ante lo que requiere quien está en proceso de aprender.

Por otra parte, este proceso depende de la calidad y pertinencia de la mediación instrumental, como sustenta Vygotsky. Depende de la mediación simbólica, representada fundamentalmente por el uso del lenguaje verbal, motor de la construcción de los procesos psicológicos superiores. A su vez, es necesario atender al concepto nuclear de esta concepción, el de Zona de Desarrollo Próximo (ZDP).

Vygotsky era consciente de que en la práctica escolar habitual era frecuente: o bien la despreocupación por el nivel de desarrollo alcanzado por el niño, de modo que la instrucción operaba fuera del alcance de éste, es decir, más allá del área de la ZDP, o bien la minusvaloración del potencial de aprendizaje del niño, de modo que la instrucción se limitaba a actuar dentro de la ZDR (Zona de Desarrollo Real), y, por tanto, no tiraba del desarrollo. El concepto de ZDP no supone, pues, sólo un deseo de un mejor pronóstico psicológico del desarrollo, sino que implica un método concreto para convertir ese pronóstico en desarrollo real a través de la educación (Álvarez \& del Rio, 1990, p. 116)

Para la aplicación operativa de la ZDP, como evaluación educativa y psicológica y como instrumento para la optimización del aprendizaje y del desarrollo, es necesario encontrar métodos adecuados que reúnan con sentido la mediación social e instrumental. De lo que se trata es de lograr que los procesos sociales inter-psicológicos, externos, lleguen a ser procesos intra-psicológicos, internos, que ayuden a la comprensión y al tratamiento externos del problema, y que ayuden después a la interiorización gradual de esa comprensión y tratamiento, "porque, como dice Emerson (1983), una ZDP es un diálogo entre el niño y su futuro, no un diálogo entre el niño y su pasado" (Álvarez et al., 1990, p. 119). 
"El aprendizaje es constructivo más que reproductivo y, primariamente, es un proceso social, cultural e interpersonal, gobernado tanto por factores sociales y situacionales como cognitivos" (Bolívar, 2009a, p. 80), sobre el cual se fundamenta el de las competencias, por lo que se focaliza ahora la atención en hacer posible que el alumnado tenga un papel activo en la enseñanza, comprometido e interesado en la construcción de significado, trabajando con sus iguales en la construcción social del conocimiento.

La competencia de aprender a aprender, según lo expuesto, tiene un indudable interés porque pone en marcha un proceso de metaconocimiento: observación del aprendizaje, para conseguir el aprendizaje significativo sobre el aprendizaje significativo. Además, es una de las formas en las que se concreta el propósito de hacer operativa la ZDP y constituye una ayuda para poder definir cómo conseguir un diálogo entre el sujeto que aprende (independientemente de su edad) y su futuro. Por este motivo, la concepción socio-constructivista es la perspectiva dominante en las orientaciones curriculares que enfatizan la importancia del contexto social y de la actividad verbal que posibilitan el diálogo entre agentes educativos y aprendiz y del sujeto que aprende sobre su propio aprendizaje.

Esta competencia está presente en los currículos de las diferentes etapas educativas. En la LOMCE (2013), a modo de ilustración, se define atendiendo a las dimensiones del conocimiento que el Currículum se propone fomentar: en la dimensión de saber, aludiendo al conocimiento de los procesos implicados en el aprendizaje, de lo que uno sabe y de lo que desconoce, de la disciplina, del contenido concreto de la tarea y de las estrategias posibles para afrontarlas; en la dimensión de saber hacer, es decir, estrategias de planificación y de resolución de tareas, de supervisión de acciones y de evaluación del proceso y del resultado que se ha llevado a cabo; y en la dimensión de saber ser, motivación por aprender, fomento de la necesidad y la curiosidad por el aprendizaje, sentirse protagonista del proceso y del resultado del aprendizaje y percibir autoeficacia y confianza en sí mismo. También se sigue este enfoque en los estudios superiores que, tomando como referencia el Proyecto Tuning Educational Structures in Europe (2002), se propone en Europa la competencia de aprender a aprender como una de las ocho competencias clave.

Como apunta Martín (2008), "la LOE recoge las propuestas de la Unión Europea y propone entre las ocho competencias básicas la de aprender a aprender, que en cierto sentido subyace a todas las otras" (p. 73). Supone una competencia metodológica, porque guía las acciones y el desarrollo de todas las otras competencias 
básicas. De hecho, el Marco Europeo de Competencias Clave (UE, 2005), considera esta competencia fundamental para el aprendizaje permanente a lo largo de la vida y en distintos contextos formales, no formales e informales:

\begin{abstract}
la habilidad para iniciar el aprendizaje y persistir en él, para organizar su propio aprendizaje y gestionar el tiempo y la información eficazmente, ya sea individualmente o en grupos. [...] conlleva ser consciente del propio proceso de aprendizaje y de las necesidades de aprendizaje de cada uno, determinar las oportunidades disponibles y ser capaz de superar los obstáculos con el fin de culminar el aprendizaje con éxito, [...] de utilizar y aplicar los nuevos conocimientos y capacidades en muy diversos contextos, como los de la vida privada y profesional y la educación y formación (p. 20).
\end{abstract}

En la misma línea de este Marco Europeo, que aporta que la motivación y la confianza son cruciales para la adquisición de esta competencia, Martín (2008) considera la competencia como desarrollo cognitivo y también emocional, y añade la relevancia de la autoestima, la aceptación del error o el esfuerzo necesario: "se trata de poner en marcha un largo proceso que permita conocerse como aprendiz, aceptarse y aprender a mejorar" (p. 74).

\title{
2.1 Las dimensiones de la competencia de aprender a aprender
}

Las habilidades de autorregulación son indispensables no solamente por los requisitos del aprendizaje permanente, sino también en la vida escolar para estructurar el proceso de aprendizaje autónoma y reflexivamente (Dettmers, 2010). De hecho, aprender a aprender no es un proceso que se desarrolle solamente por participar como aprendices en situaciones educativas varias, ni tampoco al margen de unas características específicas, sino que este proceso es constructivo, personal y social, y comporta la intervención de diferentes factores psicológicos e inter-psicológicos (cognitivos, metacognitivos, motivacionales, afectivos y relacionales) (Schunk, 2005). Siguiendo los trabajos de Zimmerman (1986), Pintrich (2000) y Boekaerts (2005), podemos señalar como elementos configuradores de la actividad de aprender a aprender las siguientes dimensiones:

- La dimensión cognitiva: planificación, organización, auto-instrucción, autocontrol y autoevaluación durante el proceso de aprendizaje. Para ello, son necesarias estrategias cognitivas que permitan obtener y procesar nuevos conocimientos y planificar y desarrollar una tarea estratégicamente, como codificar, relacionar, jerarquizar, memorizar y recuperar información (Boekaerts, 1999).

- La dimensión metacognitiva: comprender y controlar los propios procesos cognitivos y monitorizar el aprendizaje, mediante la conciencia de dichos 
procesos. Por ello, es crucial el conocimiento reflexivo i metacognitivo de elementos centrales en el aprendizaje (sobre la persona que aprende, sobre los contenidos o tareas y sobre las estrategias para aprender) y la supervisión metacognitiva del proceso de aprendizaje (la regulación y el control del aprendizaje) (Bolívar, 2009b)en cierta medida, está en la base de todas las demás. No en vano, en la propuesta de la Unión Europea, se inscriben en el marco del aprendizaje a lo largo de la vida. Es evidente que si son necesarias todas las competencias básicas para un aprendizaje a lo largo de la vida (lifelong learning. DiBenedetto \& Zimmerman (2013) recalcan su importancia en los procesos de aprendizaje, atendiendo a que la autoeficacia vincula las reacciones de autorreflexión previa a los procesos para nuevos ciclos de aprendizajes, lo que proporciona retroalimentación en el ciclo de autorregulación. De hecho, el Proyecto DeSeCo (2002) sitúa la reflexividad y el uso de destrezas metacognitivas como prerrequisitos de las competencias clave.

- La dimensión motivacional, afectiva y emocional: atribución de sentido al aprendizaje, que afecta al uso y desarrollo de los componentes cognitivos y metacognitivos. Los componentes motivacionales contribuyen a crear situaciones propicias para aprender a aprender, como la motivación intrínseca y extrínseca, las atribuciones, las creencias de control, el autoconcepto positivo, la persistencia, las percepciones sobre la autoeficacia, la autoestima, la seguridad, la frustración y la autonomía.

\subsection{Las fases de la autorregulación}

Pintrich conceptualiza el aprendizaje autorregulado como un "proceso activo y constructivo mediante el cual los estudiantes establecen metas para su aprendizaje y después supervisar, regular y controlar su cognición, motivación y el comportamiento, guiado y limitado para sus objetivos y características del contexto en el medio" (Pintrich, 2000, p. 453). Cabe resaltar, en esta línea, que la autorregulación académica se puede desarrollar (Boekaerts \& Corno, 2005; Schunk, 1996; B. J. Zimmerman, 1989), es "maleable y esencial para conseguir resultados exitosos de aprendizaje" (Dunn et al., 2012, p. 316).

Para Zimmerman, son "aquellos pensamientos generados, sentimientos y acciones que se planifican y se adaptan cíclicamente para la consecución de los objetivos personales" (2000, p. 14). Este autor lo caracteriza como dinámico y fluido, en vez de estático, y lo identifica como el "circuito de la retroalimentación" o "ciclo de autorregulación abierto", puesto que la autorreflexión sobre las acciones actuales afecta al esfuerzo posterior (Zimmerman, 1986, 1989, 2002, 2008): 
- En la forethought phase, los estudiantes preparan el escenario para el aprendizaje, valoran la tarea a realizar, de forma proactiva a fijarse objetivos y determinar un plan de acción con procesos estratégicos, lo cual se ve afectado por las construcciones de la motivación: autoeficacia, orientación de meta, creencias de control y valor de la tarea. ¿Por qué se hace? ¿Qué sentido tiene? ¿Para qué sirve?

- La performance phase contiene procesos de autoobservación y autocontrol, con los cuales se recoge la información que se utilizará para evaluar la eficacia del plan de acción determinado inicialmente y para avanzar en futuros esfuerzos de aprendizaje. Estos dos procesos de control de rendimiento se producen con esfuerzos durante el aprendizaje, que afectan tanto a la atención como a la acción. La autoobservación implica la aplicación de habilidades como el planteamiento de preguntas, la autoevaluación, el auto-registro y la monitorización metacognitiva; mientras que el autocontrol implica el autoaprendizaje, la focalización de la atención y otras estrategias (como leer, subrayar, tomar apuntes, ensayar e interrogarse uno mismo). ¿Qué operaciones debemos seguir? ¿Por qué? ¿Cómo lo estamos haciendo?

- La self-reflection phase se compone de los procesos de auto-juicio y autoreacción. ¿Qué hemos aprendido? ¿Cómo lo hemos aprendido? Esta es la fase final donde los procesos de autorreflexión implican la emisión de juicios y auto-reacciones, para alimentar de nuevo la fase de previsión, siguiendo el ciclo de autorregulación. Aquí se valora el propio rendimiento respecto a patrones de auto-orientación, a atribución de un rendimiento inferior al deseable, y se modifican tácticas en las estrategias anteriores para la siguiente tarea de aprendizaje.

Neus Sanmartí (2007) define la evaluación como el motor del aprendizaje y entiende el error como útil para regular el aprendizaje; recomienda, además, la búsqueda de estrategias para compartir con el alumnado el proceso evaluador y hacerlo partícipe y protagonista de su propio proceso de aprendizaje. Tal y como indica, aprender a evaluarse es una de las condiciones básicas para aprender a aprender, y para tener autonomía aprendiendo (en cualquier actividad); de hecho, "cuando se inicia un proceso de aprendizaje, lo más habitual es que se cometan errores, pero unos estudiantes son más capaces que otros para superarlos. La diferencia básica entre ellos es que unos saben evaluarse (regularse), mientras que los otros han desarrollado sistemas para aprender poco eficientes" (2010, p. 5).

A modo de resumen, desde un enfoque competencial, aprender a aprender supone ser capaz de movilizar conocimientos de diferentes tipos para enfrentarse a exigencias y retos de aprendizaje en contextos específicos y en situaciones de evaluación que se caracterizan por ser sistemas de actividad continua, reguladora, auténtica y participada (Mauri \& Rochera, 2010). Puesto que la evaluación por 
competencias cambia el punto de mira de lo que se quiere evaluar, obviando una mirada cuantificadora hacia lo que se espera que los estudiantes hayan aprendido; en las competencias, por el contrario, "se hace mirando al futuro: con qué grado de éxito pueden poner en práctica lo que han aprendido y aplicar sus conocimientos y habilidades en nuevos contextos" (Bolívar, 2009b, p. 6)

\section{METODOLOGÍA}

Pero, ¿Cómo enseñar a aprender a aprender? ¿Qué principios metodológicos deben seguirse? En cuanto a las orientaciones metodológicas que contribuyen al logro de la competencia de aprender a aprender, se trata de buscar las formas más adecuadas para hacer posible la interiorización del aprendizaje mediante la pertinencia de la mediación social y de la mediación instrumental, gracias al uso situado y contingente del lenguaje verbal, y de concretar esta tarea en la ZDP.

En coherencia con la definición de aprendizaje expuesta, se trata de concretar cómo los docentes implicados pueden ajustar la ayuda pedagógica, con los instrumentos verbales adecuados, para lograr que el alumnado construya su aprendizaje sobre su propio aprender, interiorizando las estrategias aportadas mediante esta ayuda pedagógica, en relación con un contenido de aprendizaje y alrededor de una tarea orientada a un objetivo (proyectarse hacia su futuro), contextualizada en una situación de comunicación determinada.

Para Martín (2008, p. 74) "enseñar a aprender a aprender significa conseguir que los alumnos y alumnas experimenten a lo largo de su escolaridad el placer que produce entender algo que antes no comprendíamos, resolver un problema que se nos resistía, sentirnos capaces en último término". Este proceso debe empezar en la educación infantil, y necesita de cada área de conocimiento para su desarrollo, es transversal en cuanto a áreas curriculares, etapas educativas, y trabajo en equipo o colaboración del profesorado. Para ello, es importante fomentar el desarrollo de capacidades de toma de conciencia sobre los propios procesos mentales y emocionales y sobre los ajenos. Martín, aporta los seis siguientes principios metodológicos (2008):

- Explorar las concepciones que estudiantes y docentes tienen sobre el aprendizaje: qué piensan que significa ser inteligente; sobre el carácter estable o modificable de la inteligencia; y sus teorías sobre el origen de las diferencias individuales. 
- Enseñar al alumnado a regular sus propios procesos de aprendizaje: planificar, supervisar y evaluar su comportamiento cuando se enfrentan a cualquier tarea escolar.

- Ayudar al alumnado a atribuir adecuadamente sus éxitos o fracasos, relacionándolos con causas que están bajo su control y que son modificables.

- Elogiar al alumnado a explicitar sus ideas y conocimientos (lenguaje oral y escrito), como medio para tomar conciencia de ellas ("habla epistémica").

- Colaborar o cooperar entre iguales para tomar conciencia de los procesos cognitivos y emocionales: ponerse de acuerdo y pensar en los objetivos; acordar y pensar sobre las estrategias y pasos; detectar errores propios y ajenos, y dar explicaciones de qué consideramos un error; llegar a soluciones compartidas, y explicar por qué son correctas.

- Realizar una evaluación formadora (autoevaluación y co-evaluación): ser capaz de identificar cuándo aprende y cuándo no, y qué es lo que le ayuda a aprender.

Desde otra lógica similar, Sanmartí (2010) expone que desarrollar la capacidad de autorregularse comporta: a) Identificar, reflexionar y compartir los objetivos de la actividad o actividades), es decir, saber para qué se hace lo que se hace, para qué sirve y con qué sentido; b) Anticipar y planificar la acción para realizar la tarea, ya que se requiere tiempo; y c) Compartir los criterios de evaluación para saber qué es lo más importante a tener presente y por qué. Así, se incluyen tanto los criterios de realización -aspectos u operaciones que se espera que el alumnado aplique-, como los criterios de resultados, es decir, la calidad con que se aplican los aspectos definidos en los criterios de realización y el grado de aceptabilidad fijado al valorar la pertinencia, completitud, precisión, volumen de conocimientos, originalidad, etc.

\subsection{Experiencias sobre aplicación de metodologías que integran la evaluación}

En educación superior, hemos explorado algunas experiencias realizadas al respecto desde la innovación educativa y desde la investigación, relacionadas entre sí y diseñadas con el propósito de vincular decididamente los procesos metodológicos de aprender a aprender con los procesos de evaluación, ya que, de acuerdo con lo expuesto anteriormente, la evaluación es clave para garantizar el diálogo del sujeto que aprende con su aprendizaje, mediante la interacción social. 
Nos referimos, por una parte, a los siguientes proyectos impulsados desde la Facultad de Educación de la Universidad de Barcelona (UB) por el grupo consolidado de Innovación docente en evaluación y tecnología GIDAT (Código: GIDUB-13/149.) dirigido por la Dra. Elena Cano: Aplicación de nuevos procesos de retroacción: del feedback al feedforward (2014-2015, Código: 2015PID-UB/004); Peer to Peer for Learning to Learn (P2P4L2L) (2016-2017, Código: 2016PID-UB/024). Y, por otra parte, a los proyectos de investigación REDICE, llevados a cabo en años consecutivos, también bajo la dirección de la Dra. Elena Cano: La evaluación de las competencias transversales de los estudiantes de maestro de la UB mediante un entorno basado en la web 2.0 (2008-2010, Código: 2016PID-UB/024); La evaluación formativa de competencias mediante blogs (2010-2012, Código: REDICE 2010 1002-04); Diseño, implementación y evaluación de propuestas de feedforward sostenible (2014-2016, Código: REDICE 16-966).

A lo largo de los cursos escolares en los que se han desarrollado los proyectos referidos, las experiencias docentes e investigadoras han asumido el reto de explorar de forma gradual:

1. La pertinencia del feedback docente para promover la autorregulación del aprendizaje del propio alumnado.

2. La pertinencia del feedback docente para promover la autorregulación del aprendizaje del propio alumnado y la explicitación del feedforward consecuente, como acción futura.

3. La pertinencia del feedback entre iguales para promover la autorregulación del aprendizaje del propio alumnado, tanto en los estudiantes evaluadores como en los estudiantes evaluados y la explicitación del feedforward consecuente, como acción futura.

Estas experiencias se han llevado a cabo en la Facultad de Educación de la UB con profesorado y alumnado del Grado de Pedagogía y del Grado de Maestro de Educación Primaria, en la Universitat Oberta de Catalunya, en la Universitat Rovira i Virgili y en la Universitat de les Illes Balears, sobre contenidos de aprendizaje de Prácticum y de Trabajo Final de Grado, entre otros. El proceso que se ha seguido en estas experiencias ha dibujado un recorrido de progresivo ajuste de la ayuda pedagógica, desde la ayuda docente a la proporcionada por los pares hasta alcanzar la autorregulación de aprender a aprender (Moral Santaella, 2008). 
En dichas experiencias, se ha partido del objetivo general de incidir en la optimización del aprendizaje mediante el fomento de la conciencia del alumnado sobre su aprendizaje, para promover así su autorregulación, mediante, en primer lugar, la explicitación del feedback docente, seguido de la explicitación, por parte del alumnado, de su percepción sobre su propio nivel actual y sobre sus decisiones de mejora. Estas dos explicitaciones han permitido visualizar la necesidad del ajuste de las intenciones, la necesidad de precisión y de ajuste del lenguaje de ambas partes. El resultado ha sido de un importante aumento de autoconciencia sobre el aprendizaje en relación con el contenido y con la tarea, pero también sobre su competencia comunicativa.

A partir de esta primera experiencia, se ha promovido la intervención del feedback entre iguales, además del feedback docente, como resultado se ha observado cómo se complementan ambos tipos de ayudas, puesto que los usos del lenguaje del feedback entre pares son más cercanos entre sí.

Finalmente, se ha realizado la experiencia de promover preferentemente el feedback mutuo entre iguales, con el objetivo de fomentar el aprendizaje por ambas partes. Por parte de los iguales proporcionadores de feedback, quienes han asumido el rol y la responsabilidad docente o de igual-experto. Esta asunción, que ha requerido distanciarse del rol estricto de iguales frente al docente, ha supuesto para los evaluadores un aumento del propio aprendizaje. A su vez, el alumnado evaluado ha percibido de forma positiva las aportaciones de sus pares, aceptando la interacción social generada en el grupo. Cada alumno/a evaluado/a, a su vez, debía explicitar qué tipo de cambios se le habían propuesto y cuáles había asumido, proyectando así su autorregulación hacia su propio futuro.

\section{RESULTADOS}

Realizado el análisis conceptual y metodológico sobre la competencia de aprender a aprender como enfoque general para las distintas etapas educativas y de forma específica para la educación superior, vamos a centrarnos ahora en la síntesis de los resultados que nos ha sido posible identificar.

En primer lugar, debemos referirnos al valor añadido que el enfoque educativo centrado en la competencia otorga al aprendizaje. Para ello, ha sido necesario exponer una definición de aprendizaje, cómo se concibe aprender desde el enfoque 
socio-constructivista. Desde este marco, hemos analizado la reconceptualización que supone aprender sobre el aprender. ¿Pero son similares estos dos momentos del aprender?

Como hemos visto, aprender en situaciones escolares educativas, se considera que consiste en atribuir significado y sentido a las experiencias y a los contenidos escolares. Entendemos, además, que el conocimiento nuevo se construye, o fructifica, sobre el conocimiento previo y que el sujeto que aprende ha de poder establecer relaciones substantivas, no arbitrarias, entre el conocimiento previo (...) y el nuevo conocimiento (Coll, 1988, 1995), mediante la interacción social entre la persona más experta y el aprendiz. Cabe añadir la importancia de la ZDP para orientarnos sobre cómo proceder y, a la vez, sobre cómo evaluar este proceso (Vigotsky, 1978).

En segundo lugar, resaltamos los retos asociados con la definición y medición conceptual del aprender a aprender. Para comprender qué significa aprender a aprender debemos recoger esta orientación teórico-metodológica y evaluativa sobre el aprendizaje desde una dimensión de observación del metaconocimiento hacia el pasado-presente y, especialmente, hacia el futuro del aprendiz consigo mismo (Álvarez \& del Rio, 1990). Por un lado, los organismos político-educativos (LOMCE, 2013; Tuning Educational Structures in Europe, 2002; Unión Europea, 2005) han comprendido y asumido este reto y han elaborado disposiciones concretas al respecto. Por otro lado, los referentes consultados han desplegado los componentes que subyacen en estos procesos psicológico-educativos, describiendo su naturaleza cognitiva, metacognitiva y emocional, sus componentes y fases en las que se despliega.

En tercer lugar, presentamos una valoración de los procesos de evaluación formativa sostenibles a través de los proyectos de investigación e innovación educativa descritos. El denominador común de todos ellos se basa en el fomento de la toma de conciencia del alumnado universitario sobre su propio nivel de aprendizaje, sus posibilidades futuras y su competencia comunicativa para poder acceder y transmitir su autorregulación reflexiva y, por lo tanto, la autoevaluación requerida. Para este fin, primero, se ha utilizado como instrumento metodológico y evaluativo la provisión de feedback por parte del profesorado y, segundo, la provisión mutua de retroalimentación entre iguales. Todo ello ha contribuido a optimizar el perfil competencial del alumnado universitario. 


\section{CONCLUSIONES Y DISCUSIÓN}

Conviene constatar que el paradigma de la competencia de aprender a aprender sigue abierto a futuras aportaciones e investigaciones. La sociedad del siglo XXI está abierta a importantes cambios y la educación no puede mantenerse al margen de la realidad social. Por ello, impulsar la competencia de aprender a aprender aconseja reflexionar previamente sobre algunos aspectos prácticos, como definir con claridad los perfiles del aprender a aprender para cada nivel educativo (desde una concepción competencial amplia y abierta). Todo ello implica, también, reflexionar acerca de los factores que influyen en las diferencias individuales de los aspectos y factores asociados a la competencia, así como concretar la relación que se establece entre esta y los conceptos relacionados. Asimismo, desde la práctica del proceso de enseñanza-aprendizaje, se recomienda repensar la programación de situaciones que promuevan la adquisición, modificación, reestructuración y automatización de la nueva información, habilidades o conocimiento; así como la participación e implicación del alumnado y su toma de consciencia de lo aprendido, sus dificultades y progresión.

El paradigma del aprendizaje permanente se asienta según el informe Delors (1994) en cuatro pilares básicos: aprender a vivir juntos, aprender a conocer, aprender a hacer y aprender a ser. Así pues, destacamos que "la introducción del aprender a aprender como una de las competencias básicas a lograr durante la escolarización, incluyendo los estudios superiores, es coherente con la idea de preparar al individuo para un mundo cambiante, competitivo, globalizado, tecnológico, donde la información fluye libremente y donde el aprendizaje debe ser una actitud vital, que acompaña a la persona durante toda su vida" (Teixidó, 2010, p. 143). Por ello, en la sociedad del conocimiento y de la información, uno de los principales objetivos de la escuela debiera ser ayudar al alumnado a convertirse en aprendices autónomos, de ahí la importancia de esta competencia: capacitar al alumnado a aprender de manera autónoma y autorregulada, para que trascienda en el ámbito educativo y se proyecte a lo largo de la vida (Bolívar, 2009a).

El EEES opta por mejorar la calidad del proceso de enseñanza-aprendizaje incidiendo en aspectos como el profesorado, los programas, los estudiantes, las infraestructuras, la evaluación, entre otros; un nuevo paradigma que implica cambios en la formación del profesorado, dirigiendo la actividad docente hacia el desarrollo de capacidades, habilidades, actitudes y valores que posibiliten la formación competencial, donde aprender a aprender es necesario para adaptarse a los cambios sociales, económicos y tecnológicos, y transformar nuestra realidad. Consecuentemente, es 
preciso indagar, reflexionar y debatir, hacia dónde se deberían canalizar y orientar nuestras acciones, en formación del profesorado, buscando un equilibrio entre calidad, capacitación y desarrollo profesional.

Ante los métodos tradicionales de transmisión de conocimientos y la enseñanza centrada en el rol docente, y no en el aprendizaje, ni en la autorregulación del mismo, la convergencia europea sitúa inequívocamente el proceso de aprendizaje como eje central del cambio metodológico. El modelo de enseñanza-aprendizaje que se propone destaca que lo importante no es lo que el profesor sabe o hace, sino lo que aprende el alumnado y cómo lo aprende. Todo ello conlleva el reto a las facultades de educación de una continua actualización científica y técnica de sus saberes, y de la adquisición de nuevas competencias profesionales como docentes y para los futuros docentes. De ahí la necesidad de que la formación del profesorado se plantee desde un proceso de desarrollo profesional continuo, que "ha de continuar a lo largo de la vida, reformulando lo adquirido en ella para solucionar problemas prácticos profesionales y de ciudadanía de manera que los futuros profesionales puedan ser eficientes en un entorno laboral y social cada vez más complejo y en continuo proceso de cambio" (Ávila Ruiz, López Atxurra, \& Fernández de Larrea, 2007, p. 14).

Finalmente, queremos insistir en la responsabilidad que recae en las facultades de educación y de formación del profesorado, como referentes en la creación de conocimiento científico, pedagógico y didáctico, para capacitar al alumnado universitario en el desarrollo de su aprender a aprender y, en especial, para la transposición didáctica, puesto que la orientación y la calidad de esta formación profesionalizadora va a repercutir en todas las demás etapas educativas.

\section{REFERENCIAS BIBLIOGRÁFICAS}

Álvarez, A., \& del Rio, P. (1990). Educación y desarrollo: La teoría de Vygotsky y la zona de desarrollo próximo. In C. Coll, J. Palacios, \& Á. Marchesi (Eds.), Desarrollo psicológico y educación II (pp. 93-119). Madrid: Alianza Editorial.

Ávila Ruiz, R. M., López Atxurra, R., \& Fernández de Larrea, E. (2007). Las competencias profesionales para la enseñanza-aprendizaje de las Ciencias Sociales ante el reto europeo y la globalización. Bilbao: Asociación Universitaria de Profesores de Didáctica de las Ciencias Sociales. Recuperado de http://www.ehu.eus/ documents/1530678/1576062/Parte20I.pdf

Boekaerts, M. (1999). Self-regulated learning: where we are today. International Journal of Educational Research, 31, 445-457. 
Boekaerts, M., \& Corno, L. (2005). Self-regulation in the classroom: A perspective on assess- ment and intervention. Applied Psychology: An International Review, 54(2), 199-231.

Bolívar, A. (2009a). Aprender a lo largo de la vida. MULTlárea, 4, 63-97. Recuperado de https://previa.uclm.es/cr/educacion/pdf/revista/4.pdf

Bolívar, A. (2009b). Competencia para el aprendizaje. In Competencia para el aprendizaje (p. 9). Santander. Recuperado de http://files.competenciasbasicas.webnode. es/200000164-1b7481c6e4/C.PLEN. V3 ANTONIO BOLIVAR.pdf

Cano, E. (2014). Análisis de las investigaciones sobre feedback: aportes para su mejora en el marco del EEES. Bordón, 66(4), 9-24. ISSN: 0210-5934.

Coll, C. (1988). Significado y sentido en el aprendizaje escolar. Reflexiones entorno al concepto de aprendizaje significativo. Infancia Y Aprendizaje, 41, 131-142.

Coll, C. (1995). La construcció del coneixement a l'escola, cap a l'elaboració d'un marc global de referencia per a l'educació escolar. In Psicologia de la Instrucció. Barcelona: Universitat Oberta de Catalunya.

Coll, C., Mauri, T., \& Rochera, M. J. (2012). La práctica de evaluación como contexto para aprender a ser un aprendiz competente. Profesorado, Revista de Currículum Y Formación Del Profesorado, 16(1), 49-59.

Council European. (2006). Recommendation of the European Parliament and the Council of 18 December 2006 on key competencies for lifelong learning. Official Journal of the European Union.

Delors, J. (1994). Los cuatro pilares de la educación. In La Educación encierra un tesoro (pp. 91-103). México: El Correo de la UNESO. Recuperado de https://www.uv.mx/ dgdaie/files/2012/11/CPP-DC-Delors-Los-Cuatro-pilares.pdf

Gobierno Vasco. Departamento de Educación Universidades e Investigación. (2012). Competencia para aprender a aprender. País Vasco. Recuperado de http:// ediagnostikoak.net/edweb/cas/materiales-informativos/ED marko teorikoak/ Aprender a aprender.pdf

DESECO - OCDE. (2002). Definition and Selection of Competencies: Theoretical and Conceptual Foundations. Summary of the final report "Key Competencies for a Successful Life and a Well-Functioning Society." Recuperado de http://www. portalstat.admin.ch/deseco/deseco finalreport summary.pdf

Dettmers, S. (2010). Effektive Hausaufgaben: Untersuchungen zu einem psychologischen Rahmenmodell. Universität Berlin.

DiBenedetto, M. K., \&Zimmerman, B. J. (2013). Construct and predictive validity of microanalytic measures of students' self-regulation of science learning. Learning and Individual Differences, 26, 30-41. https://doi.org/10.1016/j.lindif.2013.04.004

Dunn, K. E., Lo, W.-J., Mulvenon, S. W., \& Sutcliffe, R. (2012). Revisiting the Motivated Strategies for Learning Questionnaire: A Theoretical and Statistical Reevaluation of the Metacognitive Self-Regulation and Effort Regulation Subscales. 
Educational and Psychological Measurement, 72(2), 312-331. https://doi. org/10.1177/0013164411413461

Ibarra, M. S., \& González, G. (2015). Conceptos básicos en Evaluación como aprendizaje y empoderamiento en la Educación Superior. Cádiz: EVALfor - Grupo de Investigación. https://doi.org/http://dx.doi.org/10.13140/RG.2.1.5070.5686

LOMCE. (2013). Ley Orgánica 8/2013, de 9 de diciembre, para la mejora de la calidad educativa. España.

Martín, E. (2008). Aprender a aprender: clave para el aprendizaje a lo largo de la vida. CEE. Participación Educativa, 9, 72-78.

Mateo, J., \& Vlachopoulos, D. (2013). Evaluación en la universidad en el contexto de un nuevo paradigma para la educación superior. Educación XX1, 16(2), 183-208. https://doi.org/10.5944/educxx1.16.2.2639

Moral Santaella, C. (2008). Aprender a pensar-aprender a aprender. Habilidades de pensamiento y aprendizaje autorregulado. Bordón, 60(2), 123-137. ISSN: 0210-5934.

Pintrich, P. R. (2000). The Role of Goal Orientation in Self-Regulated Learning. Handbook of Self-Regulation, 451-502. https://doi.org/10.1016/B978-012109890-2/50043-3

Project Tuning. (2002). The Tuning Educational Structures in Europa Project. Recuperado de http://www.unideusto.org/tuningeu/images/stories/Publications/ENGLISH BROCHURE FOR WEBSITE.pdf

Riesco, M. (2008). El enfoque por competencias en el EEES y sus implicaciones en la enseñanza y el aprendizaje. Tendencias Pedagógicas, (13), 79-106. Recuperado de https:// revistas.uam.es/tendenciaspedagogicas/article/view/1892

Sanmartí, N. (2007). 10 ideas clave. Evaluar para aprender. (E. Graó, Ed.) (Vol. 1a). Barcelona.

Sanmartí, N. (2010). Avaluar per aprendre: I'avaluació per millorar els aprenentatges de I'alumnat en el marc del currículum per competències. Generalitat de Catalunya: Departament d'Educació, Direcció G, 36. Recuperado de http://xtec.gencat.cat/ web/.content/alfresco/d/d/workspace/SpacesStore/0024/fc53024f-626e-423b877a-932148c56075/avaluar per aprendre.pdf

Schunk, D. H. (1996). Goal and self-evaluative influences during children's cognitive skill learning. American Educational Research Journal, 33, 359-382.

Schunk, D. H. (2005). Self-Regulated Learning: The Educational Legacy of Paul R. Pintrich. Educational Psychologist, 40(2), 85-94. https://doi.org/10.1207/ $\underline{\text { s15326985ep4002 } 3}$

Teixidó, J. (2010). «Aprendre a Aprendre» a l'escola i a l'institut. Desenvolupament de la competència d'aprendre a aprendre a l'educació obligatòria. Revista Catalana de Pedagogia, 7, 137-162. https://doi.org/10.2436/20.3007.01.50

Unión Europea. (2005). Competencias clave para el aprendizaje permanente - un marco de referencia europeo. Recuperado de http://www.europarl.europa.eu/sides/getDoc. 
do?pubRef=-//EP//NONSGML+TC+P6-TC1-COD-2005-0221+0+DOC+WORD+V0// ES

Vygotsky, L. (1978). El desarrollo de los procesos psicológicos superiores. México: Grijalbo.

Zabalza, M. (2005). Guía para la planificación didáctica de la docencia universitaria en el marco del EEES. Universidad de Santiago de Compostela. Galicia. Recuperado de http://www.udc.es/grupos/apumefyr/docs/guiadeguias.pdf

Zimmerman, B. J. (1986). Becoming a self-regulated learner: Which are the key subprocesses? Contemporary Educational Psychology, 11(4), 307-313. https://doi. org/10.1016/0361-476X(86)90027-5

Zimmerman, B. J. (1989). Models of Self-regulated Learning and Academic Achievement. In B. J. Zimmerman \& D. H. Schunk (Eds.), Self-regulated Learning and Achievement: theory, research and Practice (pp. 1-25). New York: Springer_Verlag.

Zimmerman, B. J. (2000). Attaining Self-Regulation: A social cognitive perspective. In M. Boekaerts, P. R. Pintrich, \& M. Zeidner (Eds.), Handbook of Self-Regulation (pp. 13-39). San Diego, CA: Academic Press. https://doi.org/10.1016/B978$\underline{012109890-2 / 50031-7}$

Zimmerman, B. J. (2002). Becoming a Self-Regulated Learner: An Overview. Theory Into Practice, 41(August), 64-70. https://doi.org/10.1207/s15430421tip4102

Zimmerman, B. J. (2008). Darshanand Ramdass. Journal of Advanced Academics, 20(1), 18-41. https://doi.org/10.4219/jaa-2008-869 